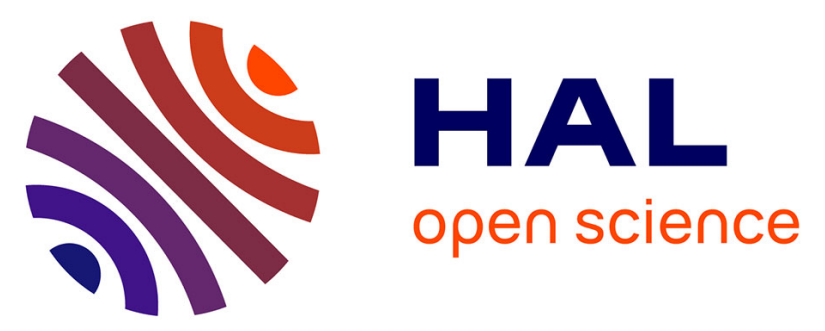

\title{
Audiogenic seizure as a model of sudden death in epilepsy: A comparative study between four inbred mouse strains from early life to adulthood
}

Benoit Martin, Gabriel Dieuset, Jodi L. Pawluski, Nathalie Costet, Arnaud Biraben

\section{To cite this version:}

Benoit Martin, Gabriel Dieuset, Jodi L. Pawluski, Nathalie Costet, Arnaud Biraben. Audiogenic seizure as a model of sudden death in epilepsy: A comparative study between four inbred mouse strains from early life to adulthood. Epilepsia, 2020, 61 (2), pp.342-349. 10.1111/epi.16432 . hal02469271

HAL Id: hal-02469271

https://hal-univ-rennes1.archives-ouvertes.fr/hal-02469271

Submitted on 24 Jun 2020

HAL is a multi-disciplinary open access archive for the deposit and dissemination of scientific research documents, whether they are published or not. The documents may come from teaching and research institutions in France or abroad, or from public or private research centers.
L'archive ouverte pluridisciplinaire HAL, est destinée au dépôt et à la diffusion de documents scientifiques de niveau recherche, publiés ou non, émanant des établissements d'enseignement et de recherche français ou étrangers, des laboratoires publics ou privés. 
Audiogenic seizure as a model of sudden death in epilepsy (SUDEP): a comparative study between four inbred mouse strains from early life to adulthood

Benoît Martin $^{1 *}$, Gabriel Dieuset ${ }^{1}$, Jodi L. Pawluski ${ }^{1}$, Nathalie Costet ${ }^{1,2}$ and Arnaud Biraben ${ }^{1}$

${ }^{1}$ Univ Rennes, CHU Rennes, Inserm, LTSI (Laboratoire de Traitement du Signal et de l'Image), UMR1099, F-35000 Rennes, France.

${ }^{2}$ Univ Rennes, Inserm, EHESP, Irset (Institut de Recherche en Santé, Environnement et Travail), UMR_S 1085, F-35000 Rennes, France

*Corresponding author: Benoît Martin, PhD, LTSI - INSERM U1099, Université de Rennes 1, Campus de Beaulieu. Bât 22, F-35000 Rennes, France, phone: +33 2232360 27, fax: +33 2232369 17, e-mail: benoit.martin@univ-rennes1.fr . Orcid ID 0000-0002-8412-1488

Keywords: mouse model, epilepsy, sudden death, audiogenic, SUDEP

Number of text pages: 17

Number of characters in the title: 149

Number of words in the abstract: 275

Number of words in the main text: 3308

Number of references: 22

Number of figures: 4

Number of tables: 0 
ABSTRACT

Objective: Mouse models of SUDEP using Audiogenic Seizures (AGS) are valuable because death can occur following a sound-induced seizure in the absence of any pharmacologic or electric component. However, only a few strains of mice are AGS prone and the vast majority of studies involve $\mathrm{DBA} / 2$ or $\mathrm{DBA} / 1$ inbred strains. With the goal to characterize the variation of AGS susceptibility with age, and to offer a larger panel of mice available for AGS studies, we performed a comparative study of the variability in AGS responses.

Methods: The variation of AGS with age was determined in two classically used inbred strains of mice, DBA/2 and DBA/1, and two additional strains, BALB/c and 129/SvTer. As AGS stimulated tonic seizures can be lethal or non-lethal, even in the same inbred strain, in a second experiment, we addressed whether there is an innate capacity to reproduce the same response after a tonic seizure in AGS, referred to as "determinism", in the DBA/2J, DBA/1J, and 129/SvTer mouse strains.

Results: Results show that the $129 /$ SvTer mouse is a more versatile model of SUDEP due its wider age range of susceptibility compared to the DBA/2J and DBA/1 J mouse strains. In addition, we show that determinism is not consistently evident in DBA/2J and 129/SvTer strains after AGS. Hence, one cannot be certain that a lethal AGS will always be lethal in a successive testing after resuscitation and vice versa in these two mouse strains.

Significance: These studies highlight the phenotypic variability of AGS in different mouse strains, show the value of an additional mouse strain, the 129/SvTer for studies using AGS, and, thus, provide valuable information for future studies of AGS and SUDEP. 


\section{INTRODUCTION}

Animal models of sudden unexpected death in epileptic patients (SUDEP) have been valuable tools used to explore the underlying mechanisms of this phenomenon in hopes of treating and preventing death due to epilepsies ${ }^{1-6}$. Typically mouse models of SUDEP use audiogenic seizures (AGS), induced with a sound stimulation, to induce $\mathrm{SUDEP}^{7}$. Immediately after the sound presentation, the mouse manifests a stereotyped behavior for which one can identify successively wild running, a clonic seizure, a tonic-clonic seizure when the mouse falls on its flanks, and a tonic seizure with an extension of the limbs toward the tail, followed, or not, by death. AGS in mice represents a valuable model for SUDEP as death can result after seizure ${ }^{7,8}$ when using non-invasive techniques without any pharmacologic or electrical component and because there is a shared respiratory mechanism of death in both SUDEP and AGS models $5,9-12$.

Only a few strains of mice are AGS prone with the vast majority of studies involving DBA/2 or, recently, DBA/1 inbred strains. For example, DBA/2 mice show AGS which appears to be age dependent with younger mice being more prone to $\mathrm{AGS}^{13}$. AGS in $\mathrm{DBA} / 1$ mice is evident from postnatal days 21 to $100^{14}$. The vast majority of the AGS studies involve the DBA/2 inbred strain simply because 1) it is an AGS responder, and 2) it is a mouse strain that is well described genetically and phenotypically. Recently the DBA/1 has been highlighted as an alternate model of AGS for its larger time frame of susceptibility, despite the lack of data about this strain compared to the DBA/2 strain. However, there are a number of emerging problems with the DBA substrains such as the decreasing AGS susceptibility with age and their fragility (ie. stunted growth) which make it difficult to do further investigations of SUDEP that would require surgical manipulations. 
Thus, with the goal to offer alternative mouse strains for AGS studies, we characterized AGS susceptibility in two strains of mice, BALB/c and 129/SvTer, that we have chosen based on the work of Fuller and Sjursen $(1967)^{15}$. We characterized the variability in AGS responses during 13 weeks in the two strains of mice, BALB/c and 129/SvTer, not previously studied in AGS beyond the work of Fuller and Sjursen (1967), as well as in two currently used inbred strains of mice for AGS, DBA/2 and DBA/1. The experiments were conducted on independent groups of mice at different ages, from days 21 to 112 of age, and for each testing time, we recorded the percentages of mice $1 /$ presenting no seizure or just wild running, 2/ presenting clonic seizures without a tonic seizure, 3 / presenting a nonlethal tonic seizure, 4/ presenting a lethal tonic seizure after AGS. None of the groups received priming. Priming is commonly used in models of AGS and represents a type of kindling where, at a critical age, a mouse is subjected to AGS during several consecutive days and then reintroduced to AGS at a later age ${ }^{6}$. This is expected to maximize the severity of the AGS response. In fact, priming can create susceptibility to AGS even in mouse strains that do not acutely respond to AGS ${ }^{2-4,16}$.

In order to study pharmacological interventions for SUDEP in mouse models, one often gives the pharmacological intervention after the mouse has been resuscitated after cardio-respiratory arrest induced by AGS and then tests the mouse for AGS response with the assumption that without the pharmacological intervention the mouse would have cardio-respiratory arrest after AGS (ie. determinism). This concept of determinism, that the same innate response will occur in response to the same stimulus under the same conditions, is often applied to lethal (experimentally a seizureinduced respiratory arrest or SIRA) and non-lethal AGS (non-SIRA) with the assumption that a SIRA is followed by a SIRA during the next test or a non-SIRA is followed by a non-SIRA during the next test. Thus, to further investigate this idea of determinism, that once a mouse experiences a SIRA, it 
will always experience a SIRA and vice versa, in a second study we tested and re-tested the same animal for SIRA or non-SIRA responses, with a resuscitating gesture after the first test if necessary.

\section{METHODS}

Animals. Male and female mice from DBA/2J, DBA/1J, BALB/cJ, and 129/SvTer strains were used in the present study. We chose the DBA/2J and DBA/1J strains because they are established models of AGS. We also chose BALB/cJ and 129/SvTer mice as comparison groups as work suggests that they present a contrasted susceptibility to $\mathrm{AGS}^{15}$. The 4 mouse strains are maintained at the University of Rennes 1 animal facility. Mice used for the present study were housed in cages of 3 to 5 individuals of the same sex and age with ad libitum access to tap water and standard mouse chow. Lights were maintained in a 12:12 hour cycle with lights on at 7:30a.m. All care and experimental manipulation of the animals were carried out in accordance with the guidelines of the European Union (directive 2010/63/EU). Weight growth curves for DBA/1J and 129/SvTer mice were generated up to the age of 100 days.

\section{Experiment 1: AGS in four strains of mice}

Each week from 21-112 days of age, an independent sample of animals averaging between 17-25 animals for each of the 4 strains was subjected to the same sound stimulus. The presentation of the sound occurred in a sound proof chamber to ensure that non-experimental animals would not be disturbed. During testing, each mouse was individually subjected to a $110 \mathrm{~dB}$ and $12 \mathrm{kHz}$ sound stimulus created by a sonicator (Deltasonic model Delta 920) in a circular plastic enclosure, $30 \mathrm{~cm}$ in diameter and $35 \mathrm{~cm}$ in height. The sound stimulus was stopped as soon as the tonic seizure appeared 
or after $60 \mathrm{sec}$ without response. We counted: a) the number of mice without seizures, b) the number of mice with clonic seizures only, c) the number of mice with a non-lethal tonic seizure, d) the number of mice with lethal tonic seizures. The results of this portion of the study are described below and presented in Figure 1.

\section{Experiment 2: An investigation of determinism}

To address the question of determinism, a further experiment took place with DBA/2Jand 129/SvTer strains. These mouse strains were subjected to AGS for days. DBA/2J mice were subjected to AGS for 2 consecutive days and 129/SvTer mice were subjected to AGS for 5 consecutive days. For this repeated AGS testing, DBA/2J mice (28-35 days of age) and 129/SvTer mice (35-70 days of age) were exposed to a sound as described above. Only mice that showed an AGS that was tonic in nature on each day of testing were included in this analysis. It should be noted that hearing loss is a common

feature with the strains of mice used in AGS studies ${ }^{17-19}$. However, hearing loss was not a concern in this experiment as only animals that responded to AGS with a tonic seizure were included in this study. Therefore, they had to hear the sound stimulation.

If an animal had cardio-respiratory arrest after seizure it was resuscitated 2-3 seconds after ear relaxation with a respirator $(1.25 \mathrm{ml}, 180 \mathrm{bpm}$; Columbus Instruments CIV-101). This resulted in a series of SIRA and non-SIRA for each mouse; 2 values for DBA/2J and 5 values for 129/SvTer mice. Considering the large percentage of DBA/2J mice not presenting with a tonic seizure as an initial AGS response at this age, which was a criterion for this experiment, a large number of animals would be needed for 5 days of testing. Thus, it was only feasible to test this strain for 2 consecutive days. Testing the $129 /$ SvTer strain for 5 consecutive days was feasible as the percentage of animals that present with 
a tonic seizure after a first AGS is high (approx. 90\%). It should also be noted that this protocol cannot be assimilated to a priming procedure since only susceptible mice, characterized by at least a tonic seizure at both test and restest, were included.

DBA/1J mice (35-42 days of age) were also used in this test-retest experiment but these mice rarely present with non-lethal tonic seizure in response to AGS (Figure 1). Effectively, to test determinism it is necessary to have mice that can present with a tonic seizure followed by death or a tonic seizure without death. Thus, interpretations with regards to determinism for DBA/1J mice are limited. . DBA/1J mice were tested on 2 consecutive days for AGS as described above. BALB/c mice were not used as they do not present with tonic seizures in response to AGS.

Statistical analyses. The determinism in the DBA/2J and 129/SvTer strains was tested by comparing the response to AGS across the first two days of testing. A Cohen's Kappa coefficient was used to test whether mice had the same response (SIRA/non-SIRA) at both times. This coefficient takes into account the possibility of a similar response between both times occurring by chance. Kappa coefficients over 0.75 are usually considered as excellent, 0.40 to 0.75 as fair to good and below 0.40 as poor. Conditional probabilities of death at day 2 (according to SIRA/non-SIRA at day 1) were produced and compared between strains using a Fisher's exact test. For the 129/SvTer strain, the same conditional probabilities were produced for the 5 consecutive days of experiment: we computed the probabilities to have a cardio-respiratory arrest after experiencing an AGS on the preceding day. A cumulative effect of repeated SIRA experiences was tested by summing the number of times through days 1 to 4 the mice had SIRA. This number was compared to the response at day 5 (SIRA/non-SIRA) using a Fisher's exact test and we computed the probability of SIRA at day 5 conditionally to the number of SIRA experiences on the preceding days. In the same way, the number of times the mouse had experienced SIRA through day 2 to day 5 was cross-tabulated according to its first response 
(SIRA/non-SIRA) at day 1 in order to assess the capacity of the response at day 1 to predict the responses on the following days. A Fisher's exact test was computed to assess statistical significance of the prediction.

To illustrate the better fitness of 129/SvTer strain to invasive experiments, we modeled the weight growth curve of 129/SvTer and DBA/2J mice from 23 and up to 88 days of age using non-linear mixed effects models, for males and females separately (random intercept, fixed effects of age and strain). The interaction of strain and age allowed for testing differential weights along the growth curve between strains.

Statistical significance was set at 5\%. Analyses were computed using the $\mathrm{SAS}^{\circledR}$ software.

\section{RESULTS}

Experiment 1. The four strains of mice presented with different responses to the AGS and differences in AGS response with age. In response to sound stimulation, the DBA/2J strain presented with 4 groups of mice characterized by their AGS response (no response, clonic seizures, non-lethal tonic seizures and lethal tonic seizures). The presence of tonic seizures after sound stimulation was very high at 21 days of age and, with age, there was a rapid extinction of AGS response in the DBA/2J strain. The DBA/1J strain at 21 days of age had a much lower susceptibility to AGS, but had a relatively stable phenotype over time with sound stimulation resulting in a majority of lethal tonic seizures or no seizure at all. In response to sound stimulation, the 129/SvTer strain presented with 3 groups of mice characterized by their AGS response (no response, non-lethal tonic seizures, lethal tonic seizures). With age, there was an increase of tonic seizure response during AGS in 129/SvTer mice. Finally, the $\mathrm{BALB} / \mathrm{cJ}$ strain showed an absence of tonic seizures (see Figure 1). 
When investigating the growth curves of DBA/1J and 129/SvTer (Figure 2), we found that 129/SvTer mice were significantly heavier than DBA/ $1 \mathrm{~J}$ mice: in males $\left(\beta_{\text {strain }}=11.7, \mathrm{p}=0.03\right.$ and interaction $\left.\beta_{\text {strain }{ }_{\text {age }}}=5.1, \mathrm{p}=0.0001\right)$ and females $\left(\beta_{\text {strain }}=11.3, \mathrm{p}=0.07\right.$ and interaction $\beta_{\text {strain }}$ age $\left.=4.9, \mathrm{p}=0.001\right)$.

Experiment 2. An investigation of the determinism in the DBA/2J, DBA/1J and 129/SvTer strains across two days of testing showed that the determinism hypothesis was rejected based on the data collected from both DBA/2J mice (kappa=0.23, Figure 3) and 129/SvTer mice (kappa=0.28, Figure 3). This result is clearly illustrated when we investigate 5 consecutive days of AGS in 129/SvTer mice where only $54.5 \%$ of mice consistently showed 5 consecutive days of SIRA or non-SIRA. However, even if one rejects the idea of determinism, we observed that in $129 / \mathrm{SvTer}$ mice the probability to have a SIRA was higher when a SIRA was experienced the day before. After daily AGS testing, the probability of a SIRA on the $5^{\text {th }}$ day of testing was correlated to the number of SIRA on the previous days (Fisher's exact test $\mathrm{p}<0.0001$, Figure 4). In addition, when looking at predictability of a SIRA or non-SIRA after the first AGS, if a mouse experienced a SIRA on the $1^{\text {st }}$ day of testing there was a $40 \%$ chance that the mouse would not have another SIRA. On the other hand, if an AGS was not lethal on the $1^{\text {st }}$ day of testing, there was a $65 \%$ chance that the mouse would not experience SIRA with additional AGS (Fisher's exact test, $\mathrm{p}<0.0001$ ). Interestingly, there were strain differences in the response to the first AGS experience such that in DBA/2J mice $80 \%$ of the mice that had a SIRA on day 1 also had a SIRA on day 2, and only $43 \%$ of mice that had a non-SIRA on day 1 had a non-SIRA on day 2 (Fisher's exact test, $\mathrm{p}=0.12$ ). In comparison, in $129 / \mathrm{SvTer}$ mice $50 \%$ of mice that had a SIRA on day 1 had a SIRA on day 2, whereas $78 \%$ of mice that had a non-SIRA on day 1 had a non-SIRA on day 2 (Fisher's exact test, $\mathrm{p}=0.21$ ). 
When testing and retesting DBA/1J mice, only 12 of 95 mice reached the criteria to be included in experiment 2; which is presenting with a tonic seizure for two consecutive days. Of these 12 mice, 11 mice exhibited a SIRA on the first and second day of testing. One mouse had a non-SIRA tonic seizure on the first day and a SIRA the second day.

\section{DISCUSSION}

There are a limited number of rat and mouse strains that can be used for studying SUDEP through AGS. However, rodent AGS models exhibit three main problems 1) rats do not die with AGS (for review see Ross and Coleman $\left.{ }^{8}\right), 2$ ) there is a small time frame in popular AGS mouse strains (DBA/2 and DBA/1) for which AGS can occur, but 3) these two inbred strains available for AGS are fragile in nature and limit the ability to perform surgical techniques since it is becoming necessary. Our goal in this study was to offer additional mouse strains for AGS studies and, thus, we characterized AGS susceptibility in four strains of mice, extending the work of Fuller and Sjursen (1967). We included the commonly used DBA/2 and DBA/1 mouse strains, as well as the less well-studied BALB/c and 129/SvTer mouse strains. Independent groups of mice were tested each week between 21-112 days of age. This was carried out in the absence of priming. We also investigated the determinism (i.e. innate consistency of the AGS response) in DBA/2 and 129/SvTer strains to address the feasibility of using case-crossover designs in pharmacological studies of SUDEP.

Indeed, with the four mouse strains investigated, DBA/2J, DBA/1J, 129/SvTer and BALB/cJ, there were different patterns in the response to AGS and in the long-term sensitivity to AGS. These results converge and extend the findings of Fuller and Sjursen (1967) who studied the AGS susceptibility of eleven mouse strains, three of which we have tested (DBA/2, 129/SvTer, BALB/c), for 21 days only, 
between ages 21 to 42 days. Here we show the progression of AGS with increasing ages, up to 112 days of age (Figure 1). We also included DBA/1 mice, which have become a common model used in AGS research since the publication of Fuller and Sjursen $(1967)^{4,14}$. In terms of the evolution of the tonic seizure response in AGS, we see an extinction of the phenotype (DBA/2), stability of the phenotype (DBA/1), increasing of the phenotype (129/SvTer) and an absence of the phenotype $(\mathrm{BALB} / \mathrm{c})$. When looking at the pattern of the tonic seizure response with AGS (lethal or non-lethal), we see that the DBA/2 and DBA/1 strain show a majority of lethal tonic seizures, whereas the 129/SvTer strain present a balanced response with both lethal and non-lethal tonic seizures.

From our results we can conclude that the 129/SvTer mouse is an advantageous model of AGS. Effectively the $129 /$ SvTer mouse 1) has a longer seizure susceptibility with age, 2) has a heavier body weights which makes it more relevant for invasive techniques, and 3) has an equivalent level of both lethal and non-lethal tonic seizures. This was found without any priming ${ }^{6}$. With a longer seizure susceptibility (which may be due to slower hearing $\operatorname{loss}^{19}$ ) and less fragility, this strain is a valuable model to be used for studies of AGS and SUDEP, especially when surgical manipulations are necessary. With equivalent response rates to AGS of lethal and non-lethal tonic seizures, the 129/SvTer strain provides one with the opportunity to investigate both the mechanism mediating lethality and the mechanism mediating survival. For example, this model could be very useful to address the role of the tonic seizure phase since it has been suggested that this later plays a significant role in SUDEP related to post-ictal generalized EEG suppression ${ }^{20,21}$.

In further investigation of the determinism of the AGS response, we showed that $\mathrm{DBA} / 2 \mathrm{~J}$ and 129/SvTer mice did not consistently reproduce the SIRA or non-SIRA response following a tonic 
seizure through additional testing. Effectively, approximately one third of the mice for both strains showed the alternative response during the second day of AGS testing (Figure 3). This suggests that case-crossover design studies should be carried out with caution as determinism is not observed in a notable number of subjects.

When testing and retesting DBA/1J mice, all the animals did exhibit a SIRA on the second day of testing. This is not a surprise as these animals rarely exhibit a non-SIRA. Using this strain of mice was not relevant to testing determinism as they did not exhibit both SIRA and non-SIRA in response to sound stimulation. This results validates previous work by Faingold and others showing that in pharmacological studies of SUDEP, where a medication to prevent SUDEP is introduced prior to the second AGS test day, the reduction of the lethality of seizures observed is, indeed, due to the pharmacological intervention in this mouse strain ${ }^{3,4,16,22}$. Since we did not address any effects of priming in the present studies, these findings may not extend to studies where priming ${ }^{14}$ has been used.

If determinism was observed, the hypothesis of genetic factors is unlikely since these mice are inbred. One must consider epigenetic factors as more probable. Besides (epi)genetic factors, we also addressed the effects of experiencing AGS in 129/SvTer mice. Figure 4 shows the distribution of the mice that had a SIRA on test-day 5 considering the number of SIRA they demonstrated over the 4 preceding test days. One can observe that the probability to die on the $5^{\text {th }}$ test day was directly correlated to the number of SIRA on previous testing days showing the experience matters and lethality begets lethality. 
In conclusion, this work highlights the value of using the 129/SvTer strain for addressing SUDEP in AGS models due to their wider age range of susceptibility and heavier body weight. We also show that experience of lethality favors lethality. We failed to show that innate determinism is present in AGS response when priming is not used. Future studies will address if the 129/SvTer strain is susceptible to priming and, if observed, if the reproducibility of the AGS response exists. 


\section{Key PoInt BoX}

- There are strain differences in AGS response with age between DAB/2J, DAB/1J, BALB/cJ and $129 /$ SvTer mice.

- Tonic seizure response to AGS with age becomes extinct in $\mathrm{DBA} / 2$, is stable in DBA/1, increases in 129/SvTer and is absent in BALB/c.

- $129 /$ SvTer is a valuable AGS model because of its long susceptibility, equal rates of lethal and non-lethal tonic seizures, and surgery compatibility.

- In the 129/SvTer models of AGS, experience of lethality favors lethality through repeated AGS testing.

- In investigating innate determinism of the AGS response, DBA/2J and 129/SvTer mice do not consistently reproduce the lethal or non-lethal AGS response. 
ACKNOWLEDGEMENTS: BM is funded by CNRS. JLP is funded by an INCR (Institut des Neurosciences Cliniques de Rennes) grant. This project was partially supported by the French Research National Agency (ANR-17-CE19-0001 project).

Disclosure OF CONFLICTS OF INTEREST: GD, NC have no conflicts of interest. JLP received an honorarium from BINC-Geneva for unrelated work. AB received subventions from Eisai, Shire, Novartis and honorarium from Novartis, UCB-Pharma, Zogenix as member of a board. BM received subventions from Eisai and UCB-Pharma.

Ethical Publication Statement: We confirm that we have read the journals position on issues involved in ethical publication and affirm that this report is consistent with those guidelines. 


\section{REFERENCES}

1. Feng HJ, Faingold CL. Abnormalities of serotonergic neurotransmission in animal models of SUDEP Epilepsy Behav. 2017 Jun;71(Pt B):174-180.

2. Faingold CL, Randall M, Kommajosyula SP. Susceptibility to seizure-induced sudden death in DBA/2 mice is altered by adenosine Epilepsy Res. 2016 Aug;124:49-54.

3. Faingold CL, Kommajosyula SP, Long X, Plath K, Randall M. Serotonin and sudden death: differential effects of serotonergic drugs on seizure-induced respiratory arrest in DBA/1 mice Epilepsy Behav. 2014 Aug;37:198-203.

4. Faingold $\mathrm{CL}$, Randall M. Effects of age, sex, and sertraline administration on seizure-induced respiratory arrest in the DBA/1 mouse model of sudden unexpected death in epilepsy (SUDEP) Epilepsy Behav. 2013 Jul;28(1):78-82.

5. $\quad$ So EL. What is known about the mechanisms underlying SUDEP? Epilepsia. 2008 Dec;49 Suppl 9:93-98.

6. Pansani AP, Colugnati DB, Scorza CA, de Almeida AC, Cavalheiro EA, Scorza FA. Furthering our understanding of SUDEP: the role of animal models Expert Rev Neurother. 2016 May;16(5):561-572.

7. Vinogradova LV. Audiogenic kindling and secondary subcortico-cortical epileptogenesis: Behavioral correlates and electrographic features Epilepsy Behav. 2017 Jun;71(Pt B):142-153.

8. Ross KC, Coleman JR. Developmental and genetic audiogenic seizure models: behavior and biological substrates Neurosci Biobehav Rev. 2000 Aug;24(6):639-653. Review

9. Ellis SP, Jr., Szabo CA. Sudden Unexpected Death in Epilepsy: Incidence, Risk Factors, and Proposed Mechanisms Am J Forensic Med Pathol. 2018 Jun;39(2):98-102.

10. Devinsky O, Hesdorffer DC, Thurman DJ, Lhatoo S, Richerson G. Sudden unexpected death in epilepsy: epidemiology, mechanisms, and prevention Lancet Neurol. 2016 Sep;15(10):1075-1088.

11. Goldman AM. Mechanisms of sudden unexplained death in epilepsy Curr Opin Neurol. 2015 Apr;28(2):166-174. Research Support, N.I.H., Extramural

Research Support, Non-U.S. Gov't

12. Damasceno DD, Savergnini SQ, Gomes ER, Guatimosim S, Ferreira AJ, Doretto MC, et al. Cardiac dysfunction in rats prone to audiogenic epileptic seizures Seizure. 2013 May;22(4):259-266.

13. De Sarro G, Russo E, Citraro R, Meldrum BS. Genetically epilepsy-prone rats (GEPRs) and DBA/2 mice: Two animal models of audiogenic reflex epilepsy for the evaluation of new generation AEDs Epilepsy Behav. 2017 Jun;71(Pt B):165173.

14. Faingold CL, Randall M, Tupal S. DBA/1 mice exhibit chronic susceptibility to audiogenic seizures followed by sudden death associated with respiratory arrest Epilepsy Behav. 2010 Apr;17(4):436-440.

15. Fuller JL, Sjursen FH, Jr. Audiogenic seizures in eleven mouse strains J Hered. 1967 May-Jun;58(3):135-140.

16. Faingold CL, Randall M, Zeng C, Peng S, Long X, Feng HJ. Serotonergic agents act on 5-HT3 receptors in the brain to block seizure-induced respiratory arrest in the DBA/1 mouse model of SUDEP Epilepsy Behav. 2016 Oct 12;64(Pt A):166-170.

17. Connelly CJ, Ryugo DK, Muniak MA. The effect of progressive hearing loss on the morphology of endbulbs of Held and bushy cells Hear Res. 2017 Jan;343:14-33.

18. Suthakar K, Ryugo DK. Descending projections from the inferior colliculus to medial olivocochlear efferents: Mice with normal hearing, early onset hearing loss, and congenital deafness Hear Res. 2017 Jan;343:34-49.

19. Turner JG, Parrish JL, Hughes LF, Toth LA, Caspary DM. Hearing in laboratory animals: strain differences and nonauditory effects of noise Comp Med. 2005 Feb;55(1):12-23.

20. Tao JX, Yung I, Lee A, Rose S, Jacobsen J, Ebersole JS. Tonic phase of a generalized convulsive seizure is an independent predictor of postictal generalized EEG suppression Epilepsia. 2013 May;54(5):858-865.

21. Rajakulendran S, Nashef L. Postictal Generalized EEG Suppression and SUDEP: A Review J Clin Neurophysiol. $2015 \mathrm{Feb} ; 32(1): 14-20$.

22. Faingold $\mathrm{CL}$, Tupal S, Randall M. Prevention of seizure-induced sudden death in a chronic SUDEP model by semichronic administration of a selective serotonin reuptake inhibitor Epilepsy Behav. 2011 Oct;22(2):186-190. 


\section{Figure legends}

Figure 1. Percentages of specific AGS responses in four inbred strains of mice, DBA/2J, DBA/1J, $\mathrm{BALB} / \mathrm{cJ}$, and 129/SvTer, between day 21 and day 112 of age. Separate groups of mice were tested at each week. TCS=tonic-clonic seizure, $\mathrm{D}=$ death, $\mathrm{S}=$ seizure, $\mathrm{TS}=$ tonic seizure. (Average $\mathrm{n}=17$ 25/group/week).

Figure 2. Weight growth curves of 129/SvTer vs DBA/1J mices (23-88 days) fitted by non-linear mixed effects models, males and females (predicted values $\pm 95 \%$ confidence intervals).

Figure 3. Percentages of mice in the DBA/2J $(n=43)$ and $129 / \operatorname{SvTer}(n=33)$ strains that experienced SIRA then SIRA, non-SIRA then non-SIRA, SIRA then non-SIRA, or non-SIRA then SIRA responses across two consecutive days of AGS testing.

Figure 4. Probability (\%) to present a respiratory arrest on test-day 5 (Y axis) according to the number of respiratory arrests demonstrated over the 4 preceding test days in $129 / \mathrm{SvTer}$ mice $(\mathrm{n}=33)$. For example, a mouse which has experienced 3 respiratory arrests among the 4 preceding testing days has a $75 \%$ probability to experience a new respiratory arrest on test-day 5 . 